$\rho=$ density of polymer particles

$\psi==\left[1+\left(D_{W} / m D_{P}\right)\right]^{-1}$

$\Phi_{M}=$ fraction for a radical of monomer or transfer agent to escape from a particle

$\Phi_{I}=$ fraction for a radical of initiator fragment to escape from a particle in the form of initiator radical $[-]$

\section{Literature cited}

1) Benngough, W. I. and H. W. Melville: Proc. Roy. Soc., A203, 429 (1955)

2) Brodnyan, J.G., J.A. Cala, T. Konen and E.L. Kelley: J. Colloid Sci., 18, 73 (1963)

3) Chung, K. H. and S. K. Han: J. Korean Chem. Soc., 6, $19(1962)$

4) French, D. M.: J. Polymer Sci., 32, 395 (1958)

5) Gerrens, S. H.: "Advances in Polymer Sci.," 1, 234 (1959)

6) Giskehang, K.: Symposium on the Chemistry of Polymerization Processes, London, April (1965)

7) Ham, G. (ed.): "Vinyl Polymerization", Part 1, Dekker, New York (1963)

8) Harada, M., M. Nomura, H. Kojima, W. Eguchi and S. Nagata: to be published

9) Harkins, W. D.: J. Amer. Chem. Soc., 69, 1428 (1947)

10) Matsumoto, M. and J. Ukida, H. Iwasaki; Kobunshi Kagaku, 7, 39 (1950)
11) Nakagawara, S.: Master Thesis, Kyoto Univ. (1969)

12) Nomura, M., M. Harada, W. Eguchi and S. Nagata; to be published

13) Okamura, S. and T. Motoyama: Mem. Fac. Eng., Kyoto Univ., 17, 220 (1955)

14) Okamura, S. and T. Motoyama: J. Polymer Sci., 58, $221(1962)$

15) O'Toole, J. T.: J. Polymer Sci., 9, 1291 (1965)

16) Parts, A. G. and D. E. Moore: J. Oil Colour Chemists Assoc., 45, 648 (1962)

17) Patsiga, R., M. Litt and V. Stannet: J. Phys. Chem. 64, $801(1960)$

18) Peggion, E., F. Testa and G. Talamini: Macromol. Chem., 71, 173 (1964)

19) Smith, W. F. and R. H. Ewart: J. Chem. Phys., 16 , 592 (1948)

20) Smith, W. F.: J. Amer. Chem. Soc., 70, 3695 (1948)

21) Stockmayer, W.H.: J. Polymer Sci., 24, 313 (1957)

22) Swain, C. G., W. H. Stockmayer and J.J. Clark: J. Am. Chem. Soc., 72, 5426 (1950)

23) Ugelstad, J., P. C. Mork and J. O. Asen: J. Polymer Sci., 5, 2281 (1967)

24) Ugelstad, J., P. C. Mork and P. Dahl: J. Polymer Sci., Part C, 27, 49 (1969)

25) Van der Hoff, B. M. E.: J. Phys. Chem., 60, 1250 (1956)

26) Vanzo, E.: Ph. D. Thesis, State Univ. College of Forestry at Syracuse Univ., Syracuse, N. Y. (1963)

\title{
THEORETICAL STUDY OF THE SCATTER OF EXPERIMENTAL DATA DUE TO PARTICLE-SIZE-DISTRIBUTION*
}

\author{
HIROAKI MASUDA AND KOICHI IINOYA \\ Department of Chemical Engineering, Kyoto University, \\ Kyoto, Japan
}

\begin{abstract}
The scatter of data due to the size distribution and the number of particles to be required in an experiment in a particulate process are discussed in detail. The process dealt with in this paper is that represented by : $y=K D_{p}^{\alpha}$. If the log-normal size distribution is valid, the number of particles required in an experiment and the amount of scatter can be determined by use of only one parameter, calculable from the standard geometric deviation of population-particles, basis number $\beta(\beta=0$ for the count basis and $\beta=3$ for the mass basis), the exponent $\alpha_{1}$ and the magnitude of error required in the experiment. Conversely, the error can be estimated from the number of particles used in an experiment.
\end{abstract}

\section{Introduction}

One of the most important subjects in the study of a particulate process is why the experimental data obtained for the process scatter more widely than in other processes. With regard to this matter, it has been said that the scatter may arise from agglomeration, adhesion, and some other causes. But there has never been any explanation that satisfactorily accounts for the cause.

* Received on April 24, 1970

Presented at the 35th Annual Meeting of the Soc. of Chem. Engrs., Japan, Arpil 3, 1970
It is sure that such complicated phenomena as agglomeration, adhesion, and others may be some of the causes of the scatter. More fundamentally, however, the scatter might be attributed to the size distribution of the particles.

Another cause of the scatter is that the mean particle diameter is often determined rather carelessly. The results scatter if studied by use of an incorrect mean particle diameter. This is not scatter in the ordinary sense. No curve fitted to these data by various curve-fitting methods represents the properties of the process.

In this study the undesirable scatter due to wrong use of the mean particle diameter is eliminated by 
use of the correct mean particle diameter. Another sort of scatter due to the size distribution is theoretically discussed. The scatter caused by the size distribution will be found negligibly small if the size distribution of sample-particles is obtained for each experiment. Usually, however, only the size distribution of population-particles is obtained. Consequently, the theoretical value of a process variable can be estimated by use of the population parameters (the mean and the variance, for example). It is expected, therefore, that the data will scatter around the estimate (the theoretical value). Practically, as will be discussed in this paper, there is some bias. It will be negligible, however, if the sample size is fairly large in number.

One of the most interesting problems here is how many particles should be sampled to attain satisfactory results. Generally, the approach to the problem is very difficult. The following discussion is, therefore, based upon the assumptions that the particle size distribution is log-normal, and that the interaction between particles is negligible. Then the experimental value $Y\left(m, s^{2}\right)$ of process variable is given by ${ }^{1}$;

$$
Y\left(m, s^{2}\right)=\bar{y}\left(m, s^{2}\right)
$$

where $\bar{y}$ is a linear estimate.

\section{Distribution of the Sample-Mean Particle Diameter}

A simple but most important process may be written in the form;

$$
y=K D_{p}^{\sim}
$$

where $K, \alpha$ : const. $\neq 0$

This paper deals only with the process represented by the above equation. If the distribution of samplemean particle diameter is obtained, the distribution of experimental data is known from the relation;

$$
Y=K \bar{D}_{p}^{\alpha}
$$

For this process, the population-mean particle diameter on $\beta$-basis (for the count basis, $\beta=0$ and for the mass basis, $\beta=3$ ) is given by the equation ${ }^{1,2)}$;

$$
\begin{aligned}
\vec{D}_{p} & =y^{-1}(\bar{y}) \\
& =\left\{\frac{1}{K} \frac{K \int D_{p}^{\beta} D_{p}^{\alpha} f\left(\ln D_{p}, \mu^{(0)}, \sigma^{2}\right) d \ln D_{p}}{\int D_{p}^{\beta} f\left(\ln D_{p}, \mu^{(0)}, \sigma^{2}\right) d \ln D_{p}}\right\}^{1 / \alpha} \\
& =\exp \left(\mu^{(0)}+c \sigma^{2}\right)
\end{aligned}
$$

where $c=\beta+\alpha / 2$.

And $\mu^{(0)}$ is the logarithmic mean diameter for the number distribution of the population and $\sigma^{2}$ is its variance;

$$
\begin{aligned}
& \mu^{(0)}=\int_{-\infty}^{\infty} \ln D_{p} f\left(\ln D_{p}, \mu^{(0)}, \sigma^{2}\right) d \ln D_{p}=\ln D_{p 50} \\
& \sigma=\ln D_{p 84}-\ln D_{p 50}
\end{aligned}
$$

On the other hand, the mean particle diameter of the random sample of size $n$ is shown by;

$$
\bar{D}_{p}=\exp \left(m+c s^{2}\right)
$$

where $m=\frac{\sum \ln D_{p}}{n}$, and $s^{2}=\frac{\sum\left(\ln D_{p}-m\right)^{2}}{n}$
It may be assumed that the sample size $n$ is sufficiently large. Then, by statistical theory ${ }^{3)}$, $\chi^{2} \equiv\left(n / \sigma^{2}\right) s^{2}$ follows a normal distribution with the mean $\nu \equiv n-1$ and the variance $2 \nu$. Therefore, the simultaneous distribution $\phi\left(m, S^{2}\right)$ of $m$ and $s^{2}$ is given by;

$$
\begin{aligned}
& \phi\left(m, s^{2}\right)=\frac{n}{\sigma^{3} \sqrt{2 \pi}} \sqrt{\frac{n}{2 \nu}} \cdot \\
& \quad \exp \left\{-\frac{n}{2 \sigma^{2}}\left(m-\mu^{(0)}\right)^{2}-\frac{1}{4 \nu}\left(\frac{n}{\sigma^{2}} s^{2}-\nu\right)^{2}\right\}
\end{aligned}
$$

The subject of this section is to seek the distribution of the mean particle diameter. It is found from Eq. (6) that the equation $\bar{D}_{p}=$ const. gives a curve in $\left(m, s^{2}\right)$-space. Now for the sake of simplicity, the natural logarithm of the mean particle diameter, $\ln \vec{D}_{p}$, is used instead of $\vec{D}_{p}$. Then the distribution of $\ln \bar{D}_{p}$ can be obtained if the simultaneous distribution of $m$ and $s^{2}$ is rewritten for that of $\ln \bar{D}_{p}$ and $s^{2}$. This will be shown in the following paragraph.

First of all, from Eq.(6), the following equation is obtained.

$$
d \ln \bar{D}_{p}=d m+c d\left(s^{2}\right)
$$

Therefore,

$$
\begin{aligned}
d\left(s^{2}\right) d m & =d\left(s^{2}\right) \Lambda d m=d\left(s^{2}\right) \Lambda d \ln \vec{D}_{p}-c d\left(s^{2}\right) \Lambda d\left(s^{2}\right) \\
& =d\left(s^{2}\right) \Lambda d \ln \vec{D}_{p}
\end{aligned}
$$

where symbol $d\left(s^{2}\right) A d m$ denotes the outer product of $d\left(s^{2}\right)$ and $d m$. From Eq.(10), the simultaneous distribution may be rewritten as follows.

$$
\begin{aligned}
1 & =\int_{-\infty}^{\infty} \int_{0}^{\infty} \phi\left(m, s^{2}\right) d\left(s^{2}\right) d m \\
& =\int_{-\infty}^{\infty}\left\{\int_{0}^{\infty} \phi\left(\ln \widetilde{D}_{p}-c s^{2}, s^{2}\right) d\left(s^{2}\right)\right\} d \ln \widetilde{D}_{p}
\end{aligned}
$$

(here symbol $A$ is omitted as usual). If a certain function $f\left(\ln \bar{D}_{p}\right)$ is defined by;

$$
f\left(\ln \breve{D}_{p}\right) \equiv \int_{0}^{\infty} \phi\left(\ln \bar{D}_{p}-c s^{2}, s^{2}\right) d\left(s^{2}\right)
$$

it is evident that the function $f\left(\ln \ddot{D}_{p}\right)$ satisfies the condition;

$$
\int_{-\infty}^{\infty} f\left(\ln \bar{D}_{p}\right) d \ln \vec{D}_{p}=1
$$

Therefore, it represents the distribution of $\ln \vec{D}_{p}$.

The above simple and definite method is the first developed to obtain the distribution of $\ln \vec{D}_{p}$, as far as the authors know. And it is the basis of the whole theory described in this paper.

Before proceeding with the problem of determining the distribution, it may be convenient to introduce a dimensionless quantity $\kappa$ by the equation;

$$
\kappa=\vec{D}_{p} / \vec{D}_{p}
$$

Then, Eq.(13) is rewritten as;

$$
\int_{-\infty}^{\infty} f\left(\ln \kappa+\ln \breve{D}_{p}\right) d \ln \kappa=1
$$

That is, the distribution $f$ is also the distribution of $\ln \kappa$, which is denoted by $f_{1 \mathrm{n} \kappa}$, namely;

$$
f_{\ln \kappa} \equiv f\left(\ln \kappa+\ln \vec{D}_{p}\right)
$$

If $k=1$, the mean particle diameter of the sample coincides with that of the population. This fact 
shows that the experimental value coincides with the theoretical value. This will be discussed in detail in the next section. It is better to use the distribution $f_{\text {In }}$ instead of $f\left(\ln \bar{D}_{p}\right)$ when comparing the experimental results with the theoretical.

Now the distribution $f_{\mathrm{In} x}$ can be obtained by carrying out the integration of Eq.(12) as follows;

$$
f_{\mathrm{ln} x}=\frac{\sqrt{n}}{\sigma \sqrt{2 \pi} \sqrt{2 c^{2} \sigma^{2}+1}} e^{-\frac{\varphi^{2}}{2}} \Phi(z)
$$

where, $\varphi \equiv \frac{\sqrt{n}\left(\ln \kappa+c \sigma^{2} / n\right)}{\sigma \sqrt{2 c^{2} \sigma^{2}+1}}$

$$
z \equiv \sqrt{\frac{2\left(2 c^{2} \sigma^{2}+1\right)}{2}}\left(2 c \ln \kappa+2 c^{2} \sigma^{2}+1\right)
$$

and $\Phi(z) \equiv \frac{1}{\sqrt{2} \pi} \int_{-\infty}^{z} e^{-\frac{z^{2}}{2}} d z$

(see also Appendix A)

This equation will prove useful when the problem "how many particles should be sampled in an experiment" is discussed.

\section{Number of Particles Required in the Experiment}

The distribution of experimental value $Y$ is obtained by use of the distribution $f_{1 \mathrm{n} \kappa}$. If $Y^{\cdot}$ denotes $Y\left(\mu^{(0)}, \sigma^{2}\right)$, ratio $\lambda$ of $Y\left(m, s^{2}\right)$ to $Y^{\cdot}$ is related to $\kappa$ by the following equation.

$$
\lambda \equiv Y / Y^{\cdot}=\left(\bar{D}_{p} / \bar{D}_{p}\right)^{\alpha}=\kappa^{\alpha}
$$

For a certain function $f_{\mathrm{in} 2}$, therefore, the following equation is set up.

$$
f_{\ln \lambda} d \ln \lambda=\alpha f_{\ln \lambda} d \ln \kappa
$$

So, if the function $f_{\ln \lambda}$ is defined by;

$$
f_{\ln \lambda} \equiv f_{\ln \alpha} / \alpha=f\left(\ln \lambda / \alpha+\ln \bar{D}_{p}\right) / \alpha
$$

it satisfies the relation;

$$
\int_{-\infty}^{\infty} f_{\ln \lambda} d \ln \lambda=1
$$

Therefore, $f_{\ln \lambda}$ represents the distribution of $\ln \lambda$.

By use of the above distribution, the probability $P(|e| \leq \delta)$ that experimental data may be in the range of relative error $-\delta$ to $+\delta$, is obtained.

The relative error is defined as;

$$
e \equiv \frac{Y-Y^{\circ}}{Y^{*}}=\lambda-1
$$

Therefore, the probability $P(|e| \leq \delta)$ can be evaluated as follows;

$$
P(|e| \leq \delta)=\int_{\ln (1-\hat{o})}^{\ln (1+\hat{\delta})} f_{\ln \lambda} d \ln \lambda=\int_{\frac{\ln (1-\delta)}{\alpha}}^{\ln (1+\hat{\alpha})} f_{\ln \alpha} d \ln \kappa
$$

When studying the problem "how many particles should be sampled to get a satisfactory result", the following assumption is acceptable.

$$
0 \leq \frac{\delta}{|\alpha|} \ll \sigma
$$

It may be assumed that $n$ is larger than 50 . Then from Eq.(16) it can be shown for the whole range of $\ln \kappa$ that (cf. Appendix B);

$$
z \geq\left\{\sqrt{2} c^{2} \sigma^{2}+1-\frac{2 \delta \sqrt{c^{2}}}{|\alpha| \sqrt{2} c^{2} \sigma^{2}+1}\right\} \sqrt{\frac{n}{2}}
$$

However, from Eq.(22), the following relation can be

obtained; $\quad \frac{\delta}{|\alpha|} \ll \sigma \leq \frac{2 c^{2} \sigma^{2}+1}{2 \sqrt{c^{2}}}$

And therefore;

$$
\frac{2 \delta \sqrt{c^{2}}}{|\alpha| \sqrt{2 c^{2} \sigma^{2}+1}} \ll \sqrt{2 c^{2} \sigma^{2}}+1
$$

Then Eq.(23) reads;

$$
z \geq \sqrt{2 c^{2} \sigma^{2}+1} \cdot \sqrt{\frac{n}{2}} \geq \sqrt{\frac{n}{2}}>5
$$

Therefore, $\phi(z) \simeq 1$.

This simplifies Eq.(21) as follows.

$$
\begin{aligned}
P & \simeq \frac{\sqrt{n}}{\sigma \sqrt{2 c^{2} \sigma^{2}+1}} \frac{1}{\sqrt{2 \pi}} \mid \int_{-\delta / \alpha}^{\delta / \alpha} e^{-\frac{\varphi^{2}}{2}} d \ln \kappa \\
& =\frac{1}{\sqrt{2 \pi}}\left\{\int_{-\infty}^{\infty} e^{-\frac{\varphi^{2}}{2}} d \varphi-\int_{-\infty}^{-|u|} e^{-\frac{\varphi^{2}}{2}} d \varphi-\int_{|u|}^{\infty} e^{-\frac{\varphi^{2}}{2}} d \varphi\right\} \\
& =1-2 \Phi(-|u|) \\
& \quad u \equiv \frac{\sqrt{n} \delta}{\alpha \sigma \sqrt{2 c^{2} \sigma^{2}+1}}
\end{aligned}
$$

where,

Another expression of Eq.(24) is;

$$
\Phi(-|u|)=\frac{1-P}{2}
$$

If $P$ is given, $u$ can be obtained from the above equation, and the number of particles $n$ is determined from Eq. (25). " $n$ " thus given is defined as "the number of particles required" and denoted by $n_{*}$. Then from Eq.(25);

$$
\log n_{*}=-2 \log \delta+\log \omega
$$$$
\text { where, } \quad \omega \equiv u^{2} \alpha^{2} \sigma^{2}\left(2 c^{2} \sigma^{2}+1\right)
$$

(see also Fig. 4)

Parameter $\omega$ is given numerically, if the probability $P$ is assigned and the variance $\sigma^{2}$ of the populationparticles, the exponent $\alpha$ of the process variable, and the basis number $\beta$ of the experiment are known. Practical use of this equation will be shown in $\$ 4$.

\section{Example}

Following example will be of help in understanding of the theory developed in the previous section.

$$
\begin{array}{ll}
\text { Example; } \\
\text { particle size } & D_{p}(50 \%)=5[\mu], D_{p}(84.13 \%)=8[\mu] \\
\text { parameters } & \mu^{(0)}=1.61\left(\mu_{g}=\ln ^{-1} \mu^{(0)}=5[\mu]\right) \\
& \sigma^{2}=0.2209\left(\sigma=0.47, \sigma_{g}=\ln ^{-1} \sigma=1.6\right)
\end{array}
$$

process variable $y=K D_{p}^{2}(\alpha=2)$

Eq. (29) represents a process that follows Stokes' law. From Eq.(4) the population-mean particle diameter $\vec{D}_{\dot{p}}$ is given by;

$$
\vec{D}_{p}=\exp (1.61+0.2209 \cdot c)
$$

Basis-numbers ' $\beta$ ' for the count and the mass are 0 and 3 , respectively. The exponent $\alpha$ is 2, and Eq. (5) gives; $\quad c^{(0)}=1$, and $c^{(3)}=4$,

denoting $c^{(0)}$ on the count basis and $c^{(3)}$, on the mass basis. From Eq. (30), therefore, the populationmean particle diameters are $\bar{D}_{p}^{(0)}=6.24[\mu]$ and $\bar{D}_{p}^{(3)}=12.1[\mu]$. On the other hand, from Eq. $(6)$ the sample-mean particle diameters are given by;

$$
\bar{D}_{p}^{(0)}=\exp \left(m+s^{2}\right) \text { and } \bar{D}_{p}^{(3)}=\exp \left(m+4 s^{2}\right)
$$




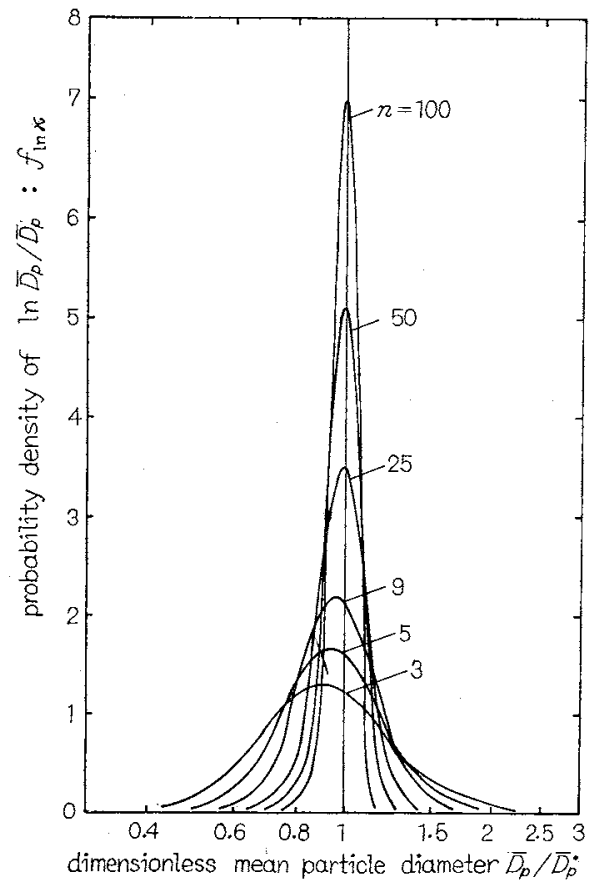

$\alpha=2, \beta=0, \sigma_{g}=1.6$

Eq. $(\mathrm{A}-10)$ is used in calculation for small $n$

Fig. 1 Distribution of $\ln \bar{D}_{p} / \bar{D}_{p}$ for the count basis

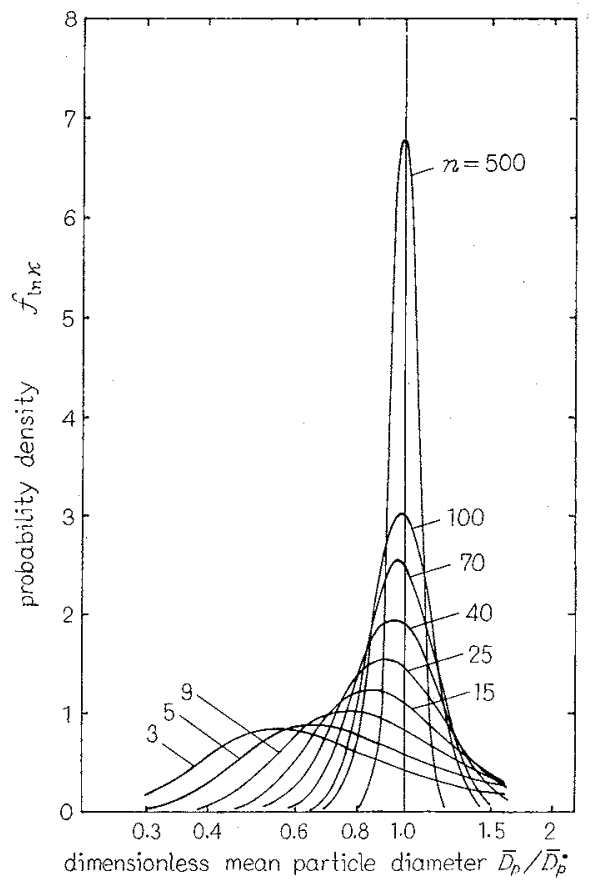

$$
\alpha=2, \beta=3, \sigma_{g}=1.6
$$

Eq. $(\mathrm{A}-10)$ is used in calculation for small $n$

Fig. 2 Distribution of $\ln \bar{D}_{p} / D_{p}$ for the mass basis

Therefore,

$$
\kappa^{(0)}=\frac{\exp \left(m+s^{2}\right)}{6.24}, \kappa^{(3)}=\frac{\exp \left(m+4 s^{2}\right)}{12.1}
$$

The distribution $f_{\ln \kappa}$ for the count and the mass bases can be obtained as shown in Figs. 1 and 2, respectively. $P(|e| \leq 0.05)$, which is the probability that the experimental data may be in the rela-

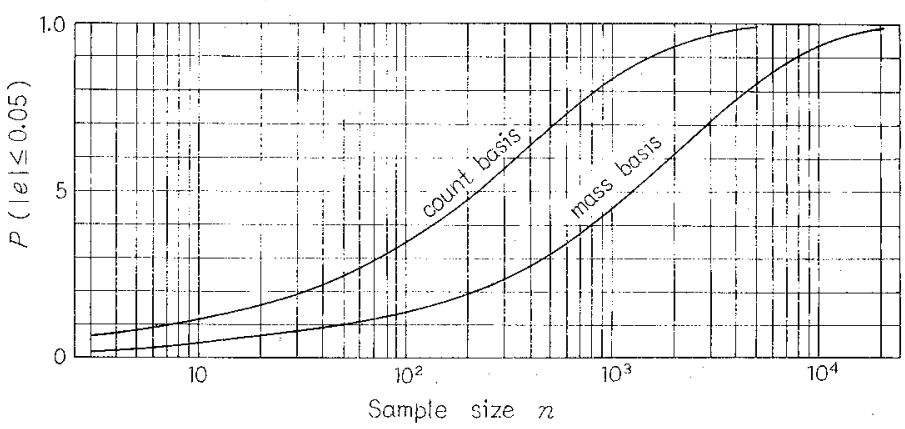

Eq. $(\mathrm{A}-10)$ is used in calculation for small $n$

Fig. 3 Probability $P(|e| \leq 0.05)$ vs. sample size $n$

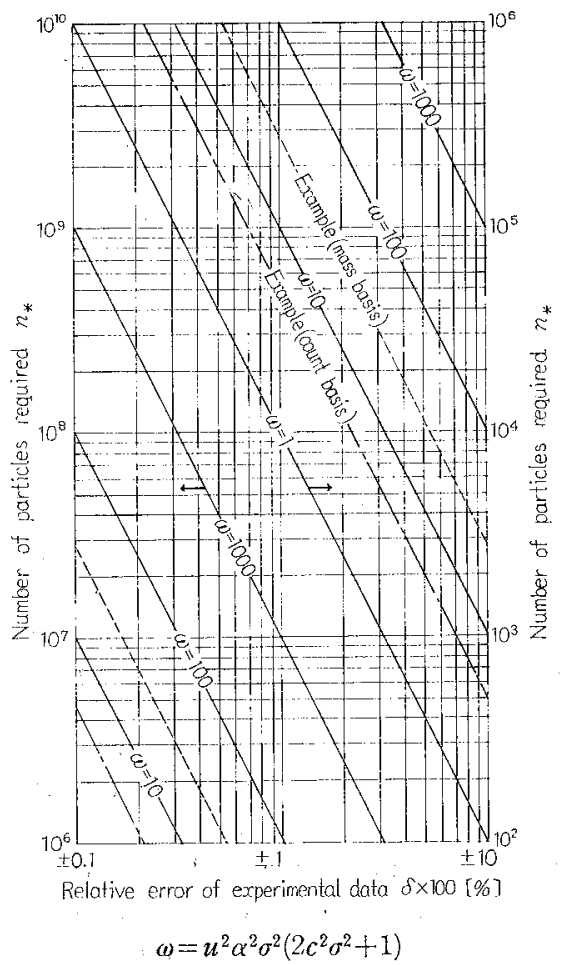

Fig. 4 Number of particles required in an experiment

tive error $\pm 5 \%$, increases as the sample size $n$ becomes larger. This is shown in Fig. 3.

The number of particles required in the experiment can be determined for the given requirement that $p \%$ of data should be in the region of relative error $\pm q \%$, where $p$ and $q$ are given numerically. In this example, the parameters $\omega$ on the count and the mass bases respectively are given as functions of $u$ as follows;

$$
\omega^{(0)}=1.27 u^{2} \text { and } \omega^{(3)}=7.13 u^{2}
$$

When $95 \%$ of the data is required to be in the region of the relative error $\pm q \%$ (where $q$ is arbitrary), for example, $u=1.96$ from Eq.(26). In this case Eq. (31) gives $\omega^{(0)}=4.87$ and $\omega^{(3)}=27.4$, as shown in Fig. 4. From the figure or from Eq.(27), "the number of particles required" is determined as follows; $n_{*}^{(0)}(95 \%, \pm 10 \%)=487, n_{*}^{(3)}(95 \%, \pm 10 \%)=2,740$, $n_{*}^{(0)}(95 \%, \pm 5 \%)=1,900, \quad n_{*}^{(3)}(95 \%, \pm 5 \%)=11,000$, 


\begin{tabular}{|c|c|c|}
\hline \multicolumn{3}{|c|}{$\begin{array}{l}\text { Table } 1 \text { Weight of particles required in the experiment } \\
\text { (cf. example in } \$ 4) \\
\qquad P(|e| \leq 0.05)=0.95, \sigma_{g}=1.6\end{array}$} \\
\hline$\sqrt[3]{\left.\sum f^{(0)} D_{p}^{3} *\right\rangle}$ & Count basis & Mass basis \\
\hline 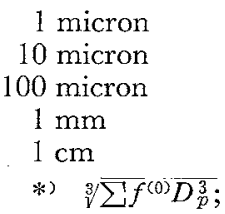 & $\begin{array}{l}2 \times 10^{-6} \mathrm{mg} \\
2 \times 10^{-3} \mathrm{mg} \\
2 \mathrm{mg} \\
2 \mathrm{~g} \\
2 \mathrm{~kg} \\
0 \mathrm{wt} \% \text { diamete }\end{array}$ & $\begin{array}{l}1.2 \times 10^{-5} \mathrm{mg} \\
1.2 \times 10^{-2} \mathrm{mg} \\
12 \mathrm{mg} \\
12 \mathrm{~g} \\
12 \mathrm{~kg}\end{array}$ \\
\hline
\end{tabular}

where $n_{*}^{(\beta)}(p \%, \pm q \%)$ denotes the number of particles required in $\beta$-basis experiment when the requirement is that $p \%$ of data should be in the region of relative error $\pm q \%$. For any probability $P, n_{*}$ will be obtained in the same manner. Just for reference, $n_{*}(95 \%, \pm 5 \%)$ is rewritten in terms of weight as specific gravity $\rho_{p}=2 \mathrm{~g} / \mathrm{cm}^{3}$ as shown in Table 1.

It is found from Fig. 4 that if 50,000 particles are used, $95 \%$ of the data will be in the region of relative error $\pm 1 \%$ on the count basis, and $\pm 2.5 \%$ on the mass basis.

\section{Discussion}

The first discussion concerns the number of particles that should be sampled to attain satisfactory results. Eq.(27) gives a part of the solution of this problem. The number of particles required is graphically shown in Fig. 4, in which $\omega$ is the only parameter. The figure shows that the number of particles required, $n_{*}$, increases with the value of $\omega$, and at the same time the scatter of the data also increases. These facts suggest that the parameter $\omega$ shows the amount of scatter. It is given by Eq. (28). From the equation it is known that the scatter of data increases as the exponent ' $\alpha$ ', variance ' $\sigma$ ' number ' $\beta$ ' increase. In the previous example $\omega^{(3)}$ was larger than $\omega^{(0)}$. Comparison between Figs. 1 and 2 shows that the scatter of the data in Fig. 2 is wider than in Fig. 1.

The parameter $\omega$ is in direct proportion to $u^{2}$ as shown in Eq. (28) or (31). And $u$ increases with $P$. Consequently, the parameter $\omega$ increases if more of the data are included in the given range of error. This fact suggests that the scatter increases relatively to the given range of error, and that $u$ shows the amount of scatter relative to the range.

From the above discussion it is found that the scatter of data is related to the standard geometric deviation $\sigma_{g}$, experimental basis - count or mass (represented by parameter $\beta$ ), and the exponent $\alpha$ which represents the process itself.

The results described above are quantitatively applicable only when the log-normal distribution is valid, but qualitatively they may apply when other distributions are used.

Our next discussion is about whether the various assumptions given in deriving Eq.(27) are appropriate or not. The extent of application of the equation will also be discussed.

One of the assumptions is that sample size $n$ is larger than 50. From the fact that particles with sample size of less than 1,000 are very rarely used in the usual experiments, this assumption is acceptable. When the sample size is small and the standard geometric deviation is larger than 2 for some reason, however, special care should be taken. As shown in Fig. 2, there is some bias in the sampling distribution of $\ln \kappa$. Practically, as one of the sampled particles is of maximum size (finite), the bias will be significant, as Mercer mentioned in his paper4). Mercer developed an adjusting method that can be applied in such a case. For adjusting by his method, however, the maximum diameter and sample median diameter have to be obtained for each experiment. Fortunately, when $\sigma_{g}$ is smaller than $1.6(\sigma<0.6)$, or the dispersion exponent for Rosin-Rammlar size distribution is larger than $2 \sim 3$, in a loose sense of the word, no special care is required about such bias. Outside of the above-mentioned range, the number of particles required is larger than $n_{*}$ which is determined by the method developed in this paper.

The next assumption given in deriving Eq.(27) is that $\delta \ll \sigma$. This assumption is easy to use, for it is independent of parameter $c$. However, it is not always necessary, because sample size $n$ is much larger than 50 . In short, the condition that parameter $z$ in Eq. (16) is larger than 5 is sufficient, because the relation, $\Phi(z) \simeq 1$ is valid. If this remark is taken into consideration, there will be no ambiguity about the assumption.

Further discussion is concerned with the assumption that the population follows the log-normal size distribution. The log-normal size distribution has various advantages which were revealed by Hatch and Choate5). In most cases, size distribution is skewed, and it can be approximated by the lognormal distribution, which is of practical use.

Only the process that is represented by Eq. (2) has been studied in this paper, but this equation can be used to represent other processes locally. The application of Eq.(27) to other processes will be therefore very interesting. The study of this possibility will be the subject of future work.

From the example in $\$ 4$, it may be said that the error due only to the size distribution is not so large as we usually experience. However, data with a little error are sometimes obtained when particles that have high fluidity are concerned. In such a case the theory developed in this study applies well and Eq.(27) is useful in examining the data. In the process where fine particles are dealt with, adhesion and agglomeration may be the principal causes. They may bring about some change in the "effective" mean particle diameter in the process. Also, the particles may interact with each other. Further investigation into these problems is necessary. 


\section{Conclusion}

In the particulate process that can be expressed by the equation $y=K D_{p}^{\alpha}$, the scatter of data caused by the size distribution increases as the standard geometric deviation ' $\sigma_{g}$ ' $\left(=\ln ^{-1} \sigma\right)$ of populationparticles, parameter ' $\beta$ ' representing the experimental basis, and exponent ' $\alpha$ ' representing the process itself, increase.

The number of particles required to get a certain per cent of the data in a certain range of error changes depending on the amount of scatter. And the number of particles required increases as the scatter increases. At the same time the number increases relatively to the magnitude of error required in the experiment. This can be represented by use of parameter $u$. When $u$ increases, the number increases too. If the log-normal size distribution is valid, all of these facts can be expressed by Eq.(27) with only one parameter $\omega=u^{2} \alpha^{2} \sigma^{2}\left(2 c^{2} \sigma^{2}+1\right)$. It is possible to use this equation in calculating the number of particles required in the experiment, and also in estimating the error when the number of particles is given.

\section{Appendix}

[A] Sampling distribution of a mean particle diameter

In the general case, simultaneous distribution is given by $^{3)}$;

$$
\phi\left(m, s^{2}\right)=\frac{\sqrt{n}}{\sigma \sqrt{2 \pi}} e^{-\frac{n}{2 \sigma^{2}}\left(\mu^{(0)}-m\right)^{2}} \cdot \frac{\left(\frac{n}{2}\right)^{\frac{n-1}{2}}}{\sigma^{2} \Gamma\left(\frac{n-1}{2}\right)}\left(\frac{s^{2}}{\sigma^{2}}\right)^{\frac{n-3}{2}} e^{-\frac{n}{2 \sigma^{2} s^{2}}}(\mathrm{~A}-1)
$$

If $n$ is odd, and can be written $n=2 k+1, k=1,2, \cdots \cdots \cdots$, the above equation reads;

$$
\phi\left(m, s^{2}\right)=\frac{\sqrt{n}\left(\frac{n}{2}\right)^{k}}{\sigma^{3} \sqrt{2 \pi} \Gamma(k)}\left(s^{2} \sigma^{k}\right)^{k-1} e^{-\frac{n}{2 \sigma^{2}}\left\{\left\langle\mu^{(0)}-m\right)^{2}+s^{2}\right\}}
$$

From Eqs. (4) and (6), we have;

$$
\begin{aligned}
& \ln \bar{D}_{p}=\mu^{(0)}+c \sigma^{2} \\
& \ln \bar{D}_{p}=m+c s^{2} \\
\therefore \quad & \ln \bar{D}_{p}^{\prime} \mid \bar{D}_{p}=\mu^{(0)}-m+c\left(\sigma^{2}-s^{2}\right)
\end{aligned}
$$

Therefore,

$$
\begin{aligned}
& -\frac{n}{2 \sigma^{2}}\left\{\left(\mu^{(0)}-m\right)^{2}+s^{2}\right\} \\
= & -\frac{n}{2 \sigma^{2}}\left(\ln \kappa+c \sigma^{2}\right)-\frac{n}{2 \sigma^{2}}\left(1-2 c \ln \kappa-2 c^{2} \sigma^{2}\right) s^{2}-\frac{n c^{2}}{2 \sigma^{2}} s^{4}
\end{aligned}
$$

If we put;

$$
s^{2}=\frac{\sigma}{\sqrt{n}=\frac{\sigma}{|c|} t} t
$$

substituting Eqs.(A-6) and (A-7) into Eq.(A-2), the following equation is obtained, by use of Eq.(12), as the sampling distribution of $\ln \kappa$

$$
f_{\mathrm{lnk}}=\frac{n^{\frac{k+1}{2}} e^{-\frac{n}{20^{2}}\left(\ln k+c a^{2}\right)^{2}}}{\sigma^{k+1} \sqrt{2 \pi} \cdot 2^{k}|c|^{k} I^{\prime}(k)} \int_{0}^{\infty} t^{k-1} \cdot e^{-x t-\frac{1}{2} t^{2}} d t
$$

where,

$$
x=\frac{\sqrt{n}}{2 \sigma|c|}\left(1-2 c \ln \kappa-2 c^{2} \sigma^{2}\right)
$$

Eq. (A -8) can be rewritten by use of Weber's function $D_{-k}(x)$ as;

$$
f_{\operatorname{In} k}=\frac{n^{\frac{k+1}{2}} e^{-\frac{n}{2 o^{2}}\left(\ln \kappa+c \sigma^{2}\right)^{2}}}{\sigma^{k+1} \sqrt{2 \pi} \cdot 2^{k} \cdot|c|^{k}} e^{\frac{x^{2}}{4}} D_{-k}(x)
$$

[B] Derivation of Eq. (23)

From Eq.(16), we have

$$
z \equiv \sqrt{\frac{n}{2\left(2 c^{2} \sigma^{2}+1\right)}}\left(2 c \ln \kappa+2 c^{2} \sigma^{2}+1\right)
$$

The range of $\ln \kappa$ is

$$
\ln (1-\delta) /|\alpha| \leq \ln \kappa \leq \ln (1+\delta) /|\alpha|
$$

This can be approximated by

$$
-\delta /|\alpha| \leq \ln \kappa \leqq \delta /|\alpha|
$$

Moreover, it can be assumed that $c$ is positive, because if $c$ is negative then the following derivation can be made over by reversing the order.

When $\ln \kappa \geq 0$, the value of $z$ satisfies the following relation, from Eq. $(\mathrm{B}-1)$;

$$
z \geq \sqrt{2 c^{2} \sigma^{2}+1} \sqrt{-\frac{n}{2}}>\sqrt{\frac{n}{2}}>5 \quad(\because n>50)
$$

On the other hand, when $-\delta /|\alpha| \leq \ln \kappa<0$, we have

$$
z \geq\left\{\sqrt{2 c^{2} \sigma^{2}+1}-\frac{2 \delta \sqrt{c^{2}}}{|\alpha| \sqrt{2 c^{2} \sigma^{2}+1}}\right\} \sqrt{\frac{n}{2}}
$$

This is Eq. (23).

\section{Acknowledgement}

We are grateful to Professor M. Ikeda (Department of Applied Mathematics and Physics, Kyoto University) for valuable suggestions concerning the mathematical treatment of this work.

\section{Nomenclature}

$$
\begin{array}{lll}
c & =\beta+\alpha / 2, \text { Eq. }(5) \\
D_{p} & =\text { particle diameter } \\
\bar{D}_{p} & =\text { sample-mean particle diameter } \\
\bar{D}_{p} & =\text { population-mean particle diameter } \\
e & =\text { relative error defined by Eq. }(20) \\
f\left(\ln \bar{D}_{p}\right) & =\text { sampling distribution of } \ln \bar{D}_{p} \\
f_{\ln \kappa} & =\text { sampling distribution of } \ln \kappa \\
K & =\text { constant, Eq. }(2) \\
m & =\text { logarithmic mean diameter for a random } \\
& \text { sample of size } n \text { (sample mean), Eq. }(7) \\
n & =\text { sample size } \\
n_{*} & \text { number of particles required in an experi- } \\
P(|e| \leq \delta)= & \text { probability that the experimental data may } \\
& \text { be in the range of relative error }-\delta \text { to }+\delta \\
p & =\text { preassigned probability } \\
q & =\text { preassigned relative error } \\
s & =\text { sample standard deviation } \\
u & =\sqrt{n} \delta /\left\{\alpha \sigma \sqrt{\left.2 c^{2} \sigma^{2}+1\right\}}\right. \\
y\left(m, s^{2}\right) & =\text { experimental value } \\
y & =\text { process variable } \\
\bar{y}\left(m, s^{2}\right) & =\text { linear estimate } \\
z & =\sqrt{\frac{n}{2\left(2 c^{2} \sigma^{2}+1\right)}} \cdot\left(2 c \ln \kappa+2 c^{2} \sigma^{2}+1\right) \\
&
\end{array}
$$

Greek letters

$$
\begin{array}{ll}
\alpha & =\text { constant, in Eq. }(2) \\
\beta & =\text { basis number }(\beta=0 \text { for the count basis, and } \\
& \beta=3 \text { for the mass basis) } \\
= & \text { relative error } \\
= & \text { dimensionless mean particle diameter of sample: } \\
= & \bar{D}_{p} / \bar{D}_{p} \\
\kappa & Y / Y=\kappa^{\alpha}, \quad \text { Eq. }(17) \\
= & \text { logarithmic mean diameter for the number } \\
\lambda & \text { distribution of population (population mean) } \\
\mu^{(0)} & \text { geometric mean particle diameter } \\
= & n-1 \\
\mu_{g} & \text { specific gravity of particle } \\
\nu & =
\end{array}
$$

VOL. 4 NO. $1 \quad 1971$ 


$$
\begin{array}{ll}
\sigma_{g} & =\text { standard geometric deviation } \\
\Phi(z) & =\text { error function } \\
\varphi & =\frac{\sqrt{n}\left(\ln \kappa+c \sigma^{2} / n\right)}{\sigma \sqrt{2 c^{2} \sigma^{2}+1}} \\
\phi\left(m, s^{2}\right) & =\text { simultaneous distribution of } m \text { and } s^{2} \\
\chi^{2} & =\left(n / \nu^{2}\right) s^{2} \\
\omega & =u^{2} \alpha^{2} \sigma^{2}\left(2 c^{2} \sigma^{2}+1\right)
\end{array}
$$

Literature cited of the 3rd Autumn Meeting of the Soc. Chem. Engrs, Japan, p. 325 (1969)

2) Chakravarti, I. M., R. G. Laha and T. Roy: "Handbook of Methods of Applied Statistics", Vol. I, p. 153, John Wiley \& Sons, Inc., New York (1967)

3) Taguchi, G.: "Jikken-keikakuhō (Design of Experiments)", Second Edition, p. 851, Maruzen, Japan (1963)

4) Mercer, T. T.: Powder Technol., 3, 65 (1969/70)

5) Hatch, T. and S. P. Choate: J. Franklin Inst., 207, $369(1929)$

1) Iinoya, K., H. Masuda and T. Kameda: Preprint

\title{
TOMOGRAPHIC OBSERVATIONS OF THE FLOW AROUND AGITATOR IMPELLER*
}

IWAO TAKASHIMA AND MASAFUMI MOCHIZUKI

Faculty of Engineering, Science University of Tokyo, Tokyo, Japan

\begin{abstract}
To make clear the local flow around each blade of an agitator, fomographic observations and measurements were carried out with a rotating camera and a plane-light source.

As the result, the profiles of local flow around an impeller look like a saw-tooth wave from a rotating coordinate projected on a horizontal plane, while the profiles of flow along top and bottom sides of each blade seems to be a pair of helicoids.

Two flow models, named vortex seet model and single vortex model are proposed to explain the action of agitator blade and the flow characteristics near the impeller.
\end{abstract}

\section{Introduction}

The flow patterns in agitated vessels have been studied by a number of researchers. However, the flow around the impeller is discussed only in a few cases $^{8)}$. Nevertheless, it is the most fundamental problem of the agitator.

The purpose of this study is to make clear the local flow around the blades of the agitator in order to propose a few desirable models of the flow for the expression of blade action by tomographic observations.

\section{Tomography for Flow around Impeller}

\subsection{Apparatus and procedure}

The experimental apparatus is shown in Fig. 1 . It consisted of a cylindrical vessel with 4 baffles, a radial flow turbine impeller in the center of the vessel, a rotating camera synchronized to the rotation of impeller, a light source, and a pair of markers. The vessel was made of clear acrylate resin and was provided with two vertical windows at a right angle

* Received on December 12, 1969

Presented at the 33th Annual Meeting of the Society of Chemical Engineers, Japan, April 3, 1968 at Kyoto each other which formed a box on the vessel wall. The box was filled with water to eliminate optical distortion. The rotating camera was a Nikon-F, whose shutter could be released by a remote controller, mounted on a turn-table driven by a geared-motor. The light source comprised a mercury lamp (DC $1 \mathrm{kw}$ ), a cylindrical lens and a slit to form a horizontal light-plane. The markers were luminescent glass tubes placed vertically near the impeller to draw a circular locus on the pictures as the basis of velocity measurements.

Fig. 2 illustrates various types of impeller employed in this experiment. The blade angle was measured by the usual method of turbomachines.

Using the light source, the rotating space of blades was cut into 9 sectional zones, as shown in Fig. 3-a. Resin particles coated with white fluorescent paint, used as tracer, were dispersed in an agitated liquid. Paths of moving particles in each lighted zone were pictured by the rotating camera at desirable time of exposure. By the rotating camera, the relative flow velocity $w$ and blade angle $\beta$ were measured as shown in Fig. 3-b. The tomograms of every zone taken by this method are seen in Fig. 4 . The length and angle of the moving paths of the particles were measured in every part of the divided space of impeller channel, as shown in Fig. 3-c. 